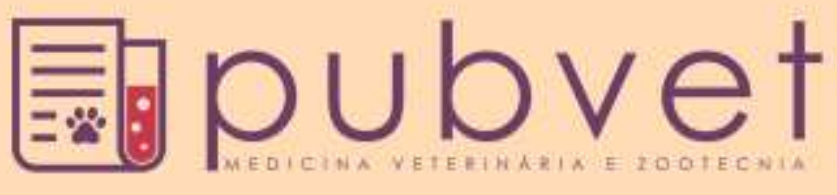

HTTP://DX.DOI.ORG/10.22256/PUBVET.V11N2.176-180

\title{
Implicações patológicas após o uso de anticoncepcional, em cadelas situadas em Teresina - PI
}

\author{
Tiago Gonçalves Azevêdo de Fonsêca Honório', Ana Paula Barros Fonseca², Estéfane Kelly \\ Dias Araújo ${ }^{1}$, Vanessa de Meneses Moura ${ }^{3}$, Rômulo Aécio Alves Chaves ${ }^{3}$, Marcelo Campos \\ Rodrigues $^{4}$, Rozeli Pizzigati Klein ${ }^{4}$
}

${ }^{\text {I}}$ Graduando em Medicina Veterinária, Universidade Federal do Piauí, Teresina, Piauí, Brasil.

${ }^{2}$ Medica Veterinária, Residente em patologia clínica, Universidade Federal do Piauí, Teresina, Piauí, Brasil.

${ }^{3}$ Médico $(a)$ veterinário $(a)$ autônomo $(a)$.

${ }^{4}$ Docente do curso de Medicina Veterinária da Universidade Federal do Piauí-Teresina-PI - Brasil.

*Autor para correspondência: E-mail: tiagohonoriof@hotmail.com

\begin{abstract}
RESUMO: As cadelas são animais multíparos de gestação curta que produzem, em geral, proles numerosas que podem atingir a maturidade sexual a partir de seis meses de idade. $\mathrm{Na}$ atualidade, o controle populacional da espécie canina e consequentemente das zoonoses deve ser contemplado em programas ou políticas públicas pelos gestores municipais e estaduais. A esterilização cirúrgica constitui a principal medida para esse controle. As drogas anticonceptivas principalmente as injetáveis para prevenir ou retardar o cio ou estro e não permitir uma fertilidade futura foram muito utilizadas na década de 80 e no início de 90 e continuam até os dias de hoje. O objetivo deste trabalho é analisar a prevalência e as principais implicações associadas ao uso de anticoncepcionais em cadelas atendidas no Hospital Veterinário Universitário "Médico Veterinário Jeremias Pereira da Silva" da Universidade Federal do Piauí, no período de janeiro de 2012 a julho de 2014, onde se pôde concluir que o uso de anticoncepcionais possivelmente está relacionado com a alta ocorrência de casos de piometra, neoplasia mamária e aborto.
\end{abstract}

Palavras chave: Aborto, anticoncepção, neoplasia, piometra

\section{Pathological implications after contraceptive use in bitches located in Teresina-PI}

ABSTRACT: Bitches are multiparous animals of short gestation, producing generally numerous offspring that can reach sexual maturity from six months old. Currently, the population control of the canine species and consequently of zoonoses must appear in programs or public policies by municipal and state managers. Surgical sterilization is the main measure for this control. The contraceptive drugs mainly injectables to prevent or slow down the heat or oestrus and not allow future fertility, were widely used in the 80s and early 90 s and continues to this day. The objective of this study is to analyze the prevalence and the main implications associated with the use of contraceptives in dogs treated at University Veterinary Hospital "Veterinarian Jeremiah Pereira da Silva" the Federal University of Piauí, from January 2012 to July 2014, where it could be concluded that the use of contraceptives is possibly related to the high incidence of cases of pyometra, breast cancer and abortion.

Keywords: Abortion, contraception, neoplasms, pyometra 


\title{
Implicaciones patológicas tras el uso de anticonceptivos en las hembras ubicadas en Teresina - PI
}

\begin{abstract}
RESUMEN. Las perras son multíparas de gestación corta que producen, generalmente, prole numerosa que puede alcanzar la madurez sexual a partir de seis meses de edad. Actualmente, el control de la población de la especie canina y consecuentemente de las zoonosis debe ser complementado en programas o políticas públicas de los gestores municipales y estatales. La esterilización quirúrgica constituye la principal medida para este control. Los medicamentos anticonceptivos inyectables principalmente para prevenir o retrasar el calor o estro y no permitir que la fertilidad futura fueron utilizados ampliamente en los años $80 \mathrm{y}$ principios de los 90 continuando hasta los días de hoy. El objetivo de este estudio es analizar la prevalencia y las principales consecuencias asociadas al uso de anticonceptivos en perras tratados en el Hospital Veterinario de la Universidad " Médico Veterinario Jeremías Pereira da Silva" de la Universidad Federal de Piauí, durante el periodo de enero de 2012 a julio de 2014, donde se puede concluir que el uso de anticonceptivos está posiblemente relacionado con la alta incidencia de casos de piómetra, cáncer de mama y aborto.
\end{abstract}

Palavras-chave: Aborto, anticoncepção, neoplasia, piometra

\section{Introdução}

Durante séculos, a exploração dos animais e da natureza pelos humanos proporcionou também aos animais de companhia a irresponsabilidade dos cuidados e da manutenção; e a procriação sem o controle devido pelos tutores ou responsáveis, propiciou condições para o crescimento dessas populações animais, gerando sérias consequências da superpopulação (Lima and Luna, 2012). Segundo o (IBGE, 2015). A população de animais de estimação em todo o mundo em nível global tem crescido No Brasil, existem cerca de 52,2 milhões de cães e 22,1 milhões de gatos.

O controle populacional de cães e gatos e consequentemente das zoonoses devem ser contemplados em programas ou políticas públicas para essas espécies como também das ações em guarda responsável, várias ações como mutirões e programas municipais para esterilização cirúrgica, como também das unidades móveis de esterilização, estão acontecendo em vários municípios brasileiros já que, frente às diversas enfermidades que podem acometê-los, torna-se importante os meios que assegurem a manutenção de sua saúde como também da sua proteção (Bittencourt, 2013).

Neste contexto, considerando que os procedimentos de contracepção de cães e gatos em programas de educação em saúde, guarda responsável e esterilização visando o controle populacional devem fazer parte de uma política de saúde pública e de bem-estar dos animais e das pessoas, o Conselho Federal de Medicina Veterinária (CFMV) publicou a Resolução ${ }^{\circ}$ 962, de 27 de agosto de 2010 normatizando tais procedimentos. $\mathrm{O}$ art. $1^{\mathrm{o}}$ dessa resolução assegura que os procedimentos contraceptivos no que tange a esterilização cirúrgica, ou não, deve oferecer ao animal o mesmo grau de eficiência, segurança e bem-estar. Nos art. $5^{\circ}$ e $6^{\circ}$, respectivamente, normatiza que os procedimentos de contracepção devem ocorrer em ambiente fechado e restrito e que os mesmos poderão ser realizados em Unidade Móvel de Esterilização e Educação em Saúde (UMEES), devidamente regularizada perante o CRMV e demais órgãos competentes.

Dessa forma, na superpopulação de cães e gatos, as medidas de controle sobre a reprodução desses animais devem receber especial atenção e a esterilização cirúrgica constitui a principal medida. No entanto, por ser um método que requer anestesia, equipamentos cirúrgicos, instalação adequada, e a presença de um médico veterinário, muitas pessoas consideram ser um procedimento oneroso, invasivo e doloroso (Cathey and Memon, 2010).

A alternativa de baixo custo é a contracepção farmacológica por meio de drogas anticonceptivas que previnem ou retardam o estro, não permitindo uma fertilidade futura. Os anticoncepcionais injetáveis foram muito utilizados na década de 80 e no início da década de 90 (Ladd et al., 1994), mas ainda nos dias de hoje podem ser encontrados 
facilmente em casas de rações e lojas do gênero, além de serem vendidos sem prescrição do médico veterinário

Uma variedade de hormônios esteroides naturais e sintéticos promove a inibição da atividade do ciclo ovariano, normalmente, transitória, mas que depende da exposição contínua à droga. Quando a administração é interrompida, o efeito da droga se dissipa e a atividade do ciclo ovariano recomeça (Feldman et al., 2014).

Entretanto, o uso de drogas contraceptivas pode ocasionar vários problemas reprodutivos na clínica de cães e gatos, podendo resultar em diversos efeitos adversos, entre eles, piometra, neoplasias mamárias e mortalidade fetal quando usado na gestação (Gabaldi, 1998, Simpson et al., 1998, Papich, 2012).

O objetivo deste trabalho é analisar a prevalência e as principais implicações associadas ao uso de anticoncepcionais em cadelas atendidas no Hospital Veterinário Universitário "Médico Veterinário Jeremias Pereira da Silva" da Universidade Federal do Piauí no período de janeiro de 2012 a julho de 2014.

\section{Material e Métodos}

Trata-se de um estudo descritivo, de corte transversal para a qual será utilizada a abordagem quantitativa com o objetivo de informar sobre a distribuição de um evento, na população, em termos quantitativos. Será analisada a relação exposiçãodoença, em uma dada população, em um particular momento fornecendo um retrato de como as variáveis estão relacionadas.

A pesquisa foi conduzida no Hospital Veterinário Universitário "Médico Veterinário Jeremias Pereira da Silva" da Universidade Federal do Piauí que dispõe de serviços de média e alta complexidade. As procedências dos animais são principalmente de proprietários que residem em Teresina-PI.

As coletas de dados foram realizadas nas fichas do arquivo do referido hospital no período de janeiro de 2012 a julho de 2014, totalizando um total de 21163 fichas. Este prontuário de atendimento contém a identificação do animal e do seu responsável, a queixa principal, sinais e sintomas, dados do exame clínico e exames laboratoriais.
Em cada ficha foram observadas e coletadas as variáveis: a informação durante a anamnese da utilização ou não do anticoncepcional e a patologia diagnosticada.

\section{Resultados e Discussão}

No levantamento das fichas segundo o acervo foram encontradas 7.765 fichas em 2012; $8.399 \mathrm{em}$ 2013 e 4.999 até julho de 2014.

Das trezentas e nove cadelas atendidas com o diagnóstico de piometra, 93 casos $(30,1 \%)$ constavam na anamnese a informação do uso de anticoncepcional (Quadro 1).

Quadro 1. Ocorrência e percentual de piometra em cadelas associada ao uso ou não de anticoncepcional, atendidas no Hospital Veterinário "Médico Veterinário Jeremias Pereira da Silva" da Universidade Federal do Piauí, no período de janeiro de 2012 a julho de 2014.

\begin{tabular}{lcc}
\hline $\begin{array}{l}\text { Referência ao uso de } \\
\text { anticoncepcional }\end{array}$ & Ocorrência & Percentual \\
\hline Usa & 93 & $30,1 \%$ \\
Não usa & 40 & $12,9 \%$ \\
Não consta & 176 & $56,9 \%$ \\
\hline Total & 309 & $100 \%$ \\
\hline
\end{tabular}

Comparando os casos de piometra em cadelas em que os responsáveis relataram o uso ou não de anticoncepcional, verifica-se que os dados vão de encontro com a literatura, como no estudo de Niskanen and Thrusfield (1998) com 953 cadelas, onde se observou que a administração do anticoncepcional aumentou em seis vezes a chance do desenvolvimento de piometra. Chen et al. (2007) e Oliveira et al. (2008) também afirmaram que o aparecimento desta afecção em cadelas tem relação com a administração dessas drogas.

A administração de anticoncepcional foi associada com esta afecção, possivelmente por potencializar os hormônios sexuais endógenos e proporcionar o relaxamento da cérvix, permitindo a entrada de bactérias da microbiota vaginal para o útero (Kutzler and Wood, 2006, Oliveira and Marques-Jr, 2006).

Em relação à neoplasia de mama 247 cadelas foram atendidas portando essa patologia, das quais, 40, ou seja, 16,2\% na anamnese havia a informação do uso de anticoncepcional (Quadro 2). 
Quadro 2. Ocorrência e percentual de neoplasia mamária em cadelas, segundo ou uso ou não de anticoncepcional, atendidas no Hospital Veterinário "Médico Veterinário Jeremias Pereira da Silva" da Universidade Federal do Piaú́, no período de janeiro de 2012 a julho de 2014.

\begin{tabular}{lcc}
\hline $\begin{array}{l}\text { Referência ao uso de } \\
\text { anticoncepcional }\end{array}$ & Ocorrência & Porcentagem \\
\hline Usa & 40 & $16,2 \%$ \\
Não usa & 42 & $16,6 \%$ \\
Não consta & 165 & $67,1 \%$ \\
\hline Total & 247 & $100 \%$ \\
\hline
\end{tabular}

É possível perceber que, nos resultados do estudo, houve equidade nos casos de neoplasia mamária quanto ao uso ou não de anticoncepcional. Pesquisadores afirmam que o número de casos da doença aumenta quando o animal é exposto ao contraceptivo (Lana et al., 2007, Sorenmo, 2003).

Das 124 fichas de cadelas gestantes que foram atendidas durante $o$ período da pesquisa apresentando sinais de aborto e confirmado na ultrassonografia a morte fetal, em $34(27,4 \%)$ havia o relato da aplicação do anticoncepcional (Quadro 3 ), alegando os tutores que desconheciam que as mesmas estavam gestantes.

Sabe-se que os métodos farmacológicos de anticoncepção podem suprimir a fertilidade pela inibição da secreção dos hormônios gonadotróficos, prevenir o desenvolvimento embrionário, interferir na atividade cíclica ou induzir a perda embrionária ou fetal (Feldman et al., 2014), fato este que explica perfeitamente e justifica a morte fetal e consequentemente o aborto.

Quadro 3. Ocorrência e percentual de aborto de cadelas segundo o uso ou não de anticoncepcional, atendidas no Hospital Veterinário "Médico Veterinário Jeremias Pereira da Silva" da Universidade Federal do Piauí, no período de janeiro de 2012 a julho de 2014.

\begin{tabular}{lcc}
\hline $\begin{array}{l}\text { Referência ao uso de } \\
\text { anticoncepcional }\end{array}$ & Ocorrência & Porcentagem \\
\hline Usa & 34 & $27,4 \%$ \\
Não usa & 22 & $17,7 \%$ \\
Não consta & 68 & $54,8 \%$ \\
\hline Total & 124 & $100 \%$ \\
\hline
\end{tabular}

\section{Conclusão}

Pôde-se concluir que o uso de anticoncepcionais em cadelas possivelmente está relacionado com a alta ocorrência de casos de piometra e tem relação com casos de neoplasia mamária e aborto.

Alerta-se para o fato que a esterilização cirúrgica é o que resulta em benefício para o controle da natalidade e na prevenção de enfermidades como a piometra e a neoplasia de mama. Neste contexto, esta pesquisa agrega que a droga anticonceptiva utilizada desde a década de 80 , não proporcionou o controle populacional e ainda predispõe a enfermidades cuja terapia é cirúrgica e com risco de morte.

Espera-se que, com os resultados do presente estudo, sejam tomadas medidas que envolvam mais segurança na promoção, proteção, recuperação e manutenção da saúde dos animais e que sejam analisados com maior rigor os casos em que se optam pelo método da contracepção hormonal nos animais, tendo em vista os riscos, muitas vezes irreparáveis, a que se predispõem à saúde dos mesmos.

\section{Referências Bibliográficas:}

Bittencourt, C. R., Souza, N. T. F., Braga, K. F., Biondo, A. W., Ribas, C. R. \& Guérios, S. D. 2013. Unidade Móvel de Esterilização e Educação em Saúde no ano de 2012. In: 34 Congresso Brasileiro da Anclivepa, Rio Grande do Norte. CD dos Anais do CBA.

Cathey, M. \& Memon, M. A. 2010. Nonsurgical methods of contraception in dogs and cats: Where are we now? Vetinary Medicine, 105, 1217.

Chen, R. F. F., Addeo, P. M. D. \& Sasaki, A. Y. 2007. Piometra aberta em uma cadela de 10 meses. Revista Acadêmica, 5, 317-322.

Feldman, E. C., Nelson, R. W., Reusch, C. \& ScottMoncrieff, J. C. 2014. Canine and feline endocrinology. Elsevier Health Sciences, Philadelphia.

Gabaldi, S. H. 1998. Hiperplasia e prolapso vaginal em cadelas. Clínica Veterinária, 17-18.

IBGE, 2015. Instituto Brasileiro de Geografia e Estatística. Mercado PET. Disponível em: <http://www.agricultura.gov.br/arq editor/file/c amaras_tematicas/Insumos_agropecuarios/79R O/IBGE PAEB.pdf. Acesso em: 10 nov. 2015. 
Kutzler, M. \& Wood, A. 2006. Non-surgical methods of contraception and sterilization. Theriogenology, 66, 514-525.

Ladd, A., Tsong, Y. Y., Walfield, A. M. \& Thau, R. 1994. Development of an antifertility vaccine for pets based on active immunization against luteinizing hormone-releasing hormone. Biology of Reproduction, 51, 1076-1083.

Lana, S. E., Rutteman, G. R. \& Withrow, S. J. 2007. Tumors of the mammary gland. Small animal clinical oncology, 4, 619-636.

Lima, A. F. M. \& Luna, S. P. L. 2012. Algumas causas e consequências da superpopulação canina e felina: acaso ou descaso? Revista de Educação Continuada em Medicina Veterinária e Zootecnia, 10, 32-38.

Niskanen, M. \& Thrusfield, M. V. 1998. Associations between age, parity, hormonal therapy and breed, and pyometra in Finnish dogs. Veterinary Record, 143, 493-498.

Oliveira, E. C. S. \& Marques-Jr, A. P. 2006. Endocrinologia reprodutiva e controle da fertilidade da cadela. Revista Brasileira de Reprodução Animal, 30, 11-18.

Oliveira, N. G., Koshyama, M. H. I., Cristina, S., Scandura, M. A. B., Leme, F. F., Lúcia, M.,
Marcucci, M. L. G. L. \& Oliveira, P. C. 2008. Uso de aglepristone e cloprostenol no tratamento de piometra em cadela - relato de caso. Unifeob. São João da Boa Vista

Papich, M. G. 2012. Manual Saunders de Terapia Veterinária. Elsevier Health Sciences Brazil.

Simpson, G., England, G. C. W. \& Harvey, M. 1998. BSAVA manual of small animal reproduction and neonatology. British Small Animal Veterinary Association, London.

Sorenmo, K. 2003. Canine mammary gland tumors. Veterinary Clinics of North America: Small Animal Practice, 33, 573-596.

\section{Article History:}

Received 6 November 2016

Accepted 25 November 2016

Available on line 2 February 2017

License information: This is an open-access article distributed under the terms of the Creative Commons Attribution License 4.0, which permits unrestricted use, distribution, and reproduction in any medium, provided the original work is properly cited. 